\title{
Corticotropin-Releasing Factor Produces Fear-Enhancing and Behavioral Activating Effects Following Infusion into the Locus Coeruleus
}

\author{
Pamela D. Butler, ${ }^{1}$ Jay M. Weiss, ${ }^{1}$ Julie C. Stout, ${ }^{2}$ and Charles B. Nemeroff ${ }^{1,3}$ \\ Departments of 'Psychiatry, ${ }^{2}$ Psychology, and ${ }^{3}$ Pharmacology, Duke University Medical Center, Durham, North Carolina \\ 27710
}

\begin{abstract}
The present series of experiments tested the hypothesis that the behavioral activating and anxiogenic effects produced by intraventricular administration of corticotropin-releasing factor (CRF) may be mediated by noradrenergic neurons in the brain-stem locus coeruleus (LC). Results showed that infusion of CRF into the LC (100 ng) significantly increased nonambulatory spontaneous motor activity measured in photocell cages; ambulatory (i.e., locomotor) activity was not altered. In the modified Porsolt swim test, which examines arousal and agitation in a stressful situation, significant behavioral activation (i.e., decreased floating) was seen following infusion of CRF (10 $\mathrm{ng}$ ) into the LC; a $500 \mathrm{ng}$ dose of CRF was necessary to produce similar effects following infusion into the lateral ventricle. The results of these 2 tests suggest that the behavioral activating effects of CRF in the LC may be related to arousing or stress-related effects, rather than to increased locomotor activity per se. Anxiogenic activity was assessed in animals placed in an open field containing a small, darkened compartment. Infusion of CRF into the LC (1-100 $\mathrm{ng})$ significantly increased the time spent in the compartment and decreased the amount of time spent exploring the outside of the compartment or venturing into the inner squares of the open field, all indices of anxiogenic behavior. Biochemical studies showed that bilateral infusion of CRF into the LC produced significant increases in the concentration of the norepinephrine metabolite 3,4-dihydroxyphenylglycol in such forebrain projection areas of the LC as the amygdala and posterior hypothalamus. These data, taken together, suggest that CRF produces its behavioral activating and anxiogenic effects, at least in part, by increasing the activity of $L C$ noradrenergic neurons.
\end{abstract}

Corticotropin-releasing factor (CRF) is a 41 -amino acid peptide (Vale et al., 1981) with a wide CNS distribution (Swanson et al., 1983; Merchenthaler, 1984). CRF stimulates the release of

\footnotetext{
Received Mar. 6, 1989; revised July 5, 1989; accepted July 5, 1989.

This work was supported by grants NIMH MH-42088 to C.B.N., NIMH MH40406 to J.M.W., and a fellowship from the National Alliance for Research on Schizophrenia and Depression (NARSAD) to P.D.B. P.D.B. was also supported by NIMH MH-15177. We are grateful to James Ritchie for assay of plasma corticosterone. We also wish to thank Dr. Clinton Kilts for his invaluable assistance in setting up the DHPG assay and Michael Johnson for expert technical assistance.

Correspondence should be addressed to Charles B. Nemeroff, M.D., Ph.D., Department of Psychiatry, Box 3859, Duke University Medical Center, Durham, NC 27710

Copyright (c) 1990 Society for Neuroscience $0270-6474 / 90 / 010176-08 \$ 02.00 / 0$
}

adrenocorticotropin hormone (ACTH) and $\beta$-endorphin from the anterior pituitary gland (Rivicr ct al., 1982), and when infused directly into the CNS, appears to mediate and coordinate autonomic (Brown and Fisher, 1985) and behavioral (Morley and Levine, 1982; Sutton et al., 1982; Sirinathsinghji et al., 1983) responses to stress. For instance, intracerebroventricular (ICV) administration of CRF produces behavioral activation in a familiar environment (Sutton et al., 1982) and produces behavior indicative of enhanced fear in a novel open field (Britton et al., 1982), an acoustic startle test (Swerdlow et al., 1986), a social interaction test (Dunn and File, 1987), and in operant conflict and conditioned emotional response paradigms (Britton et al., 1985; Cole and Koob, 1988). These locomotor-activating and anxiogenic effects of CRF appear to be independent of activation of the hypothalamic-pituitary-adrenal (HPA) axis as they are produced by central but not peripheral administration of CRF (Sutton et al., 1982; Takahashi et al., 1989) and are not blocked by hypophysectomy (Eaves et al., 1985) or by dexamethasone pretreatment (Britton et al., 1986a, b).

The CNS site(s) of action of CRF in producing these behavioral activating and anxiogenic effects are unclear. However, several studies suggest that these effects of CRF may be produced by interactions with noradrenergic (NE) neurons: ICV infusion of CRF increases NE turnover in several forebrain areas (Dunn and Berridge, 1987), CRF-induced suppression of a conditioned emotional response can be blocked by the $\beta$-adrenergic receptor antagonist propranolol (Cole and Koob, 1988), and NE receptor antagonists have been found to alter CRF-induced increases in locomotor activity (Imaki et al., 1987; Cole and Koob, 1988).

In the present series of studies, we tested the hypothesis that the brain-stem locus coeruleus (LC) may be involved in the behavioral activating and anxiogenic effects of CRF. The LC was of interest for several reasons. First, it contains the greatest number of NE cell bodies in the CNS (Foote et al., 1983) and has been repeatedly implicated in stress responses, fear, and arousal functions (Thierry et al., 1968; Korf et al., 1973; Glavin, 1985; Svensson, 1987). Second, CRF-containing neurons are in close anatomical proximity to NE neurons in the LC (Swanson et al., 1983). Third, CRF dose-dependently increases the firing rate of LC-NE neurons (Valentino et al., 1983). Finally, we have recently shown that stress, which activates NE neurons, markedly increases CRF concentrations in the LC (Chappell et al., 1986).

The effects of direct bilateral infusion of CRF into the LC were examined on spontaneous motor activity in animals adapted to photocell cages and in the modified Porsolt swim test-a 
test which is highly sensitive to arousal and agitation (Weiss et al., 1986). Anxiogenic effects were examined using a defensive withdrawal paradigm which has been shown to be sensitive to the fear-enhancing effects of ICV infusion of CRF (Takahashi et al., 1989). Parallel studies were conducted to biochemically estimate the activity of NE neurons in LC projection fields following CRF infusion into the LC.

\section{Materials and Methods}

\section{Animals}

Male Long-Evans rats (Blue Spruce Farms, Altamont, NY), weighing 250-300 gm at the start of the experiment, were housed singly with food and water available ad libitum in an environmentally controlled animal facility ( $12 \mathrm{hr}$ light/dark cycles with lights on at 0700$)$. Animals were handled daily to minimize the effects of nonspecific stress and of isolation.

\section{Surgery and intracerebral microinjections}

Rats were anesthetized with sodium pentobarbital $(50 \mathrm{mg} / \mathrm{kg}$, IP) and mounted in a Kopf stereotaxic instrument with the incisor bar set at $+5 \mathrm{~mm}$ above the interaural line. Rats were cannulated in 1 of 3 locations: guide cannulae (26 gauge; Plastic Products, Roanoke, VA) were unilaterally implanted $1 \mathrm{~mm}$ above the lateral ventricle $(\mathrm{A} / \mathrm{P},-0.5 \mathrm{~mm}$ from bregma; $\mathrm{M} / \mathrm{L}, 1.3 \mathrm{~mm} ; \mathrm{D} / \mathrm{V}, 3.5 \mathrm{~mm}$ from the surface of the skull), $2 \mathrm{~mm}$ above the cerebral aqueduct (A/P, $-0.6 \mathrm{~mm}$; D/V,$+6.2 \mathrm{~mm}$, relative to the interaural line; $\mathrm{M} / \mathrm{L}, 0 \mathrm{~mm}$ from midline), or bilaterally $3 \mathrm{~mm}$ above the $\mathrm{LC}(\mathrm{A} / \mathrm{P},-2.1 \mathrm{~mm} ; \mathrm{D} / \mathrm{V},+5.2 \mathrm{~mm}$, relative to the interaural line, $M / L, \pm 1.1 \mathrm{~mm}$ from midline). The cannulae were fixed to the skull with 4 stainless steel screws and dental cement. Following surgery, 33-gauge obturators were placed into the guide cannulae and rats were allowed to recover for at least 1 week.

Rat/human CRF (Bachem, Torrance, CA) was dissolved in artificial cerebrospinal fluid (CSF) added to $0.01 \mathrm{~N}$ acetic acid. The vehicle solution also contained $0.01 \mathrm{~N}$ acetic acid to which artificial CSF was added. In these experiments, artificial CSF rather than normal saline was used as a vehicle because infusion of normal saline alters the firing rate of LC neurons (P. E. Simson and J. M. Weiss, unpublished observations). Microinfusions were made using 33-gauge internal cannulae (Plastic Products) precut to extend 1,2 , or $3 \mathrm{~mm}$ below the ends of guide cannulae implanted in the lateral ventricle, cerebral aqueduct, or $\mathrm{LC}$, respectively. The internal cannulae were connected to a $10 \mu \mathrm{l}$ syringe with PE-20 tubing and CRF was infused in a volume of $3 \mu \mathrm{l}$ into the lateral ventricle, $1.6 \mu \mathrm{l}$ into the cerebral aqueduct, and $0.8 \mu \mathrm{l} /$ side into the LC, over a period of $2 \mathrm{~min}$. The internal cannulae were left in place for a further $30 \mathrm{sec}$ to prevent efflux and the obturators were then replaced.

\section{Motor activity}

Test 1 . Spontaneous motor activity was measured in Lucite activity cages $(43 \times 43 \times 21 \mathrm{~cm})$, equipped with 15 photocells spaced $2.5 \mathrm{~cm}$ apart across the long axis of 2 opposing sides of the box (Opto-Varimex, Columbus Instruments, Columbus, $\mathrm{OH}$ ) as previously described (Skoog et al., 1986). Rats were habituated to the cages for $4 \mathrm{hr}$ on the day prior to testing and for $2 \mathrm{hr}$ immediately prior to infusion of CRF $(0.01-1.0$ $\mu \mathrm{g}$ ) or vehicic into the ccrcbral aqucduct or LC, which always occurred in the morning at the same time $( \pm 1 \mathrm{hr})$ in the circadian cycle. Immediately following infusion, rats were returned to the same photocell cages and cumulative horizontal and ambulatory activity were recorded on a PC 900 computer (Columbus Instruments) at 15 min intervals for $120 \mathrm{~min}$. Horizontal movements were counted by the interruption of any photocell beam, whereas ambulatory (i.e., locomotor) movements were recorded by interruption of 2 adjacent photocell beams in rapid succession. Nonambulatory, or nonlocomotor, movements were defined as the horizontal minus the ambulatory counts. Animals were tested only once in this paradigm, after which they were re-randomized for dose and tested for defensive withdrawal behavior and plasma corticosterone concentrations, with at least 1 week separating each measure.

Test 2. Motor behavior was also examined in the modified Porsolt swim test (Weiss et al., 1986). At 10 or $45 \mathrm{~min}$ following infusion of CRF $(0.01-10.0 \mu \mathrm{g})$ or vehicle into the $\mathrm{LC}$ or lateral ventricle, rats were placed in a clear Plexiglas tank filled with $25^{\circ} \mathrm{C}$ water $(37.5 \mathrm{~cm}$ deep $)$. In order to prevent the animals from sinking if they ceased activity, a plastic flotation bubble was taped just behind the head of the animal; the bubble did not hinder activity but prevented the animal from sinking if it ceased to move its limbs, thereby permitting floating (immobility) to occur uninterrupted. The number of seconds spent floating or struggling were recorded for $15 \mathrm{~min}$ by an observer unaware of the dose administered. Struggling was defined as swimming vigorously so that all 4 limbs were moving and the 2 front limbs were breaking the surface of the water. Floating was defined as the time the animal remained with all 4 limbs motionless. The remaining activity could be described as swimming or treading water, but this was not measured directly. Animals used in this paradigm received only one swim test each and were not subsequently used for any other biochemical or behavioral measure.

\section{Defensive withdrawal test}

A defensive-withdrawal paradigm which has recently been shown to be sensitive to the anxiogenic effects of ICV infusion of CRF was used (Takahashi et al., 1989). Testing was conducted in an opaque Plexiglas open field $(100 \times 100 \times 50 \mathrm{~cm})$, the floor of which was marked into $20 \times 20 \mathrm{~cm}$ grids and illuminated by a fluorescent lamp. A cylindrical galvanized chamber, open at one end and measuring $15 \mathrm{~cm}$ deep and $12.5 \mathrm{~cm}$ in diameter, was secured to the floor of the open field next to the wall in a lengthwise direction $30 \mathrm{~cm}$ away from a corner of the open field. Rats received 2 consecutive days of testing. On day 1 , rats were habituated to the open field not containing the chamber for $10 \mathrm{~min}$. This first, or habituation, day was necessary because it was found that untreated animals would not emerge from the chamber unless they had been previously exposed to the open field (Takahashi et al., 1989; P. D. Butler, J. M. Weiss, J. C. Stout, and C. B. Nemeroff, unpublished observations), and thus the anxiogenic effects of CRF could not be assessed. On day 2, $45 \mathrm{~min}$ following infusion of CRF $(0.001-1.0 \mu \mathrm{g})$ or vehicle into the cerebral aqueduct or LC, animals were placed in the chamber in the open field. The length of time spent in the chamber, defined as all 4 paws in the chamber, the length of time spent exploring the outside of the chamber, defined as physical contact with or sniffing the outside of the chamber, the number of inner crossings, outer crossings, and the number of free and wall rears were recorded for $15 \mathrm{~min}$ by an observer unaware of the dose administered. The open field and chamber were cleaned with a dilute acetic acid solution between each subject to prevent olfactory cues from affecting the behavior of subsequently tested rats. The 45 min postinfusion time was chosen for testing defensive-withdrawal behavior because results in the swim test were found to be as robust at $45 \mathrm{~min}$ as at $10 \mathrm{~min}$ following infusion, and it was of interest to determine whether significant effects could be seen 45 min following infusion. Each animal was tested only once in the defensive withdrawal paradigm, and, as stated above, animals were subsequently re-randomized for dose and used for measurement of plasma corticosterone concentrations after at least a 1 week interval.

\section{Assay for NE, DHPG, DA, and DOPAC}

3,4-Dihydroxyphenylglycol (DHPG) concentration was used as an index of NE neuronal activity. While 3-methoxy,4-hydroxyphenylglycol (MHPG) is frequently used to assess NE activity, bioformation of DHPG also appears to represent a major route of NE metabolism in the CNS (Braestrup et al., 1974; Li et al., 1981, 1983) and, like MHPG, reflects changes in the functional activity of NE neurons in the rat brain (Warsh et al., 1981; Scatton, 1982).

Animals used for assay of DHPG were used in this paradigm only. Rats were decapitated 45 min following infusion of CRF $(1.0 \mu \mathrm{g} ; n=$ $8)$ or vehicle $(n=9)$ into the LC. The brain structures of interest were dissected according to the procedure of Heffner et al. (1980). Brains were sectioned into approximately 1.2 -mm-thick coronal slices using a cooled aluminum brain holder machined with blade guides from which the amygdala, posterior hypothalamus, and bed nucleus of the stria terminalis (BNST) were dissected using a no. 12 flat edge scalpel blade. Tissue samples were placed into $500 \mu$ l polypropylene microcentrifuge tubes and stored at $-70^{\circ} \mathrm{C}$ until assayed.

Frozen tissue samples were homogenized by ultrasonic cell disruption in $420 \mu \mathrm{l} 0.2 \mathrm{~N} \mathrm{HClO}_{4}$ containing $0.5 \mathrm{~mm}$ EDTA, $0.5 \mathrm{~mm}$ sodium metabisulfite, and $8.4 \mathrm{ng} \mathrm{3,4-dihydroxybenzoic} \mathrm{acid} \mathrm{(internal} \mathrm{standard).}$ Homogenates were centrifuged $(10,000 \times g$ for $10 \mathrm{~min})$ and a $200 \mu \mathrm{l}$ aliquot of the supernatant was assayed directly for free DHPG, NE, DOPAC, and DA content. Because the majority of DHPG exists in sulfoconjugated form (Li et al., 1981), we also measured total (free plus sulfoconjugated) concentrations of DHPG, NE, DOPAC, and DA. Thus, 


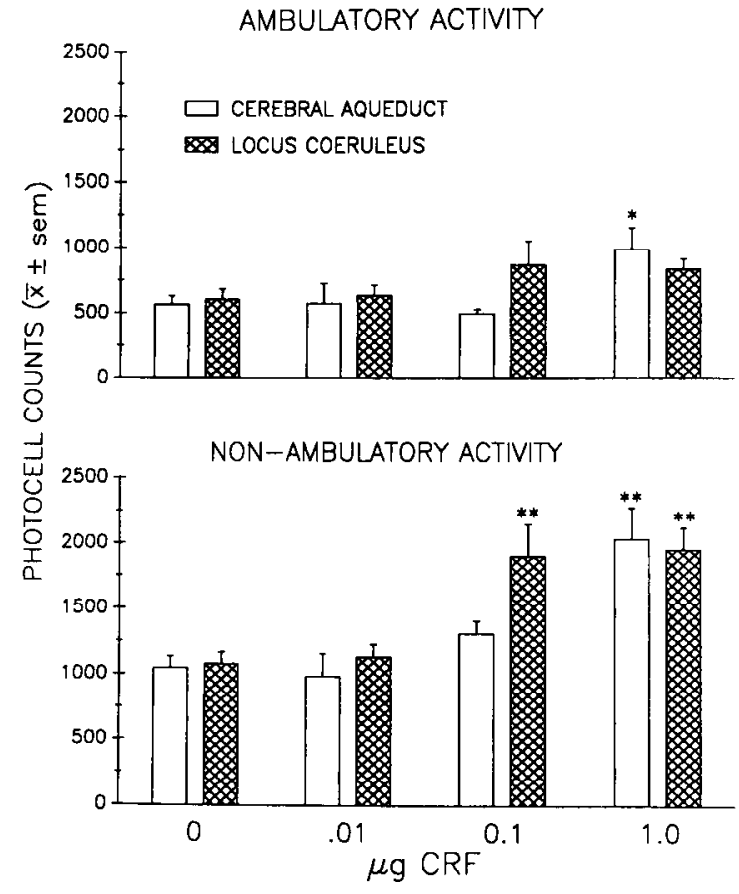

Figure 1. Effects of infusion of CRF into the cerebral aqueduct and locus coeruleus on spontaneous ambulatory and nonambulatory motor activity measured in photocell cages over a 2 -hr period ( $n=7-9$ animals in each drug group at each injection site). Significantly different from controls using Dunnett's test, ${ }^{*} p<0.05,{ }^{* *} p<0.001$.

an additional $200 \mu \mathrm{l}$ aliquot was subjected to enzymatic hydrolysis by addition of $0.8 \mathrm{mg}$ purified aryl sulfate (type H-5, Sigma, St. Louis, $\mathrm{MO}$ ) in $500 \mu \mathrm{l}$ of $0.5 \mathrm{M}$ sodium acetate buffer (pH 5.9), and samples were sealed, vortexed, and incubated for $3 \mathrm{hr}$ in a $37^{\circ} \mathrm{C}$ water bath. To the $200 \mu \mathrm{l}$ aliquots of unreacted supernatants or the reacted aliquots of unreacted supernatants or the reacted enzyme/buffer/sample mixture were added $400 \mu \mathrm{l}$ of $1 \mathrm{~m}$ Tris (pH 8.6) and approximately $15 \mathrm{mg}$ of acid-washed, heat-activated aluminum oxide. Samples were agitated in a wrist action shaker for $12 \mathrm{~min}$ followed by brief centrifugation to pellet the aluminum oxide. The sample/buffer solution was aspirated, and the aluminum oxide pellet washed twice with $1 \mathrm{ml}$ of $5 \mathrm{~mm}$ Tris (pH 8.6) with gentle vortexing and brief centrifugation, and the buffer was subsequently aspirated. Catechols were eluted by the addition of $150 \mu 10.2$ $\mathrm{N} \mathrm{HClO}_{4}$ containing $0.5 \mathrm{mM}$ EDTA and shaking $(12 \mathrm{~min})$ followed by brief centrifugation. Samples were injected in a $100 \mu \mathrm{l}$ volume. Tissue pellets were assayed for protein content by the method of Lowry et al. (1951).

Separations were performed via ion pair, reverse-phase HPLC using a stainless steel column $(250 \times 4.6 \mathrm{~mm})$ packed with octadecyl bonded microparticulate $(5 \mu \mathrm{m})$ silica (Alltech Applied Sciences, Avondale, PA). The mobile phase consisted of $0.1 \mathrm{M} \mathrm{NaH}_{2} \mathrm{PO}_{4}, 0.05 \mathrm{M}$ citrate, $0.25 \mathrm{mM}$ sodium octanesulfonate, $0.05 \mathrm{~mm}$ EDTA adjusted to a final pH of 4.0 and containing $1.56 \%$ acetonitrile. Compounds of interest were detected amperometrically following their electrochemical oxidation at a glassy carbon electrode potential of $+600 \mathrm{mV}$. The concentrations of DHPG, NE, DA, and DOPAC in tissue samples were determined by reference to a calibration curve generated by adding varying amounts of DHPG (0.05-2 ng), NE, and DA (0.25-10 ng), DOPAC (0.1-4 ng), and a constant amount ( $2 \mathrm{ng}$ ) of 3,4-dihydroxybenzoic acid to $200 \mu \mathrm{l}$ aliquots of $0.2 \mathrm{M} \mathrm{HClO}_{4}$ containing $0.5 \mathrm{~mm}$ EDTA and $0.5 \mathrm{~mm}$ sodium metabisulfite.

\section{Corticosterone assay}

CRF (0.01-1.0 $\mu \mathrm{g})$ or vehicle was infused into rats implanted with cannulae in the cerebral aqueduct or LC. Rats were killed by decapitation $45 \mathrm{~min}$ later, with care being taken to minimize stress as previously described (Uphouse et al., 1982). Trunk blood was collected in heparinized tubes and centrifuged at $1500 \mathrm{rpm}$ at $4^{\circ} \mathrm{C}$ for $10 \mathrm{~min}$, and plasma was removed and stored at $-70^{\circ} \mathrm{C}$. Plasma corticosterone concentrations were determined using a modification (Ritchie et al., 1985) of a

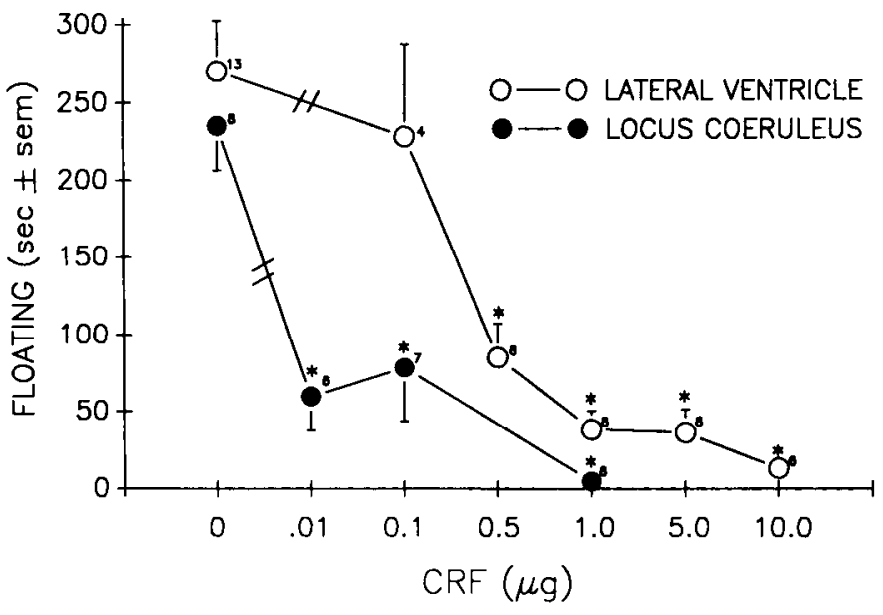

Figure 2. Time spent floating (sec $\pm \mathrm{SEM}$ ) in a 15 -min swim test 45 min following infusion of CRF into the lateral ventricle or locus coeruleus. The number of animals/group is indicated at each data point. Time spent struggling was not affected (data not shown). Significantly different from controls using Dunnett's test, ${ }^{*} p<0.001$.

competitive protein-binding radioassay (Murphy, 1967), with a sensitivity of $3.0 \mathrm{ng} / \mathrm{ml}$. Sample radioactivity was determined using a Packard 460 liquid scintillation counter.

\section{Histology}

Cannulae placements were verified with dye infusions. Rats used in the swim test only received dye infusions ( $1 \%$ thionin) and were killed by decapitation. Those receiving defensive-withdrawal and photocell activity tests followed by collection of trunk blood to measure plasma corticosterone were given dye infusions following decapitation. Brains were removed and frozen, and coronal sections cut with a microtome. Sections were thaw-mounted on glass slides and examined for dye placement. Only those rats with correct dye localization (i.e., in the lateral ventricle, cerebral aqueduct, or bilaterally in the LC) were included in the data analysis. For photographic purposes (Fig. 1), a hematoxylin and eosin stain was used to visualize the site of injection.

\section{Data analysis}

A 1 -way analysis of variance (ANOVA) was used to analyze the behavioral data, and a 2-way ANOVA was used to analyze the corticosterone data. If the ANOVA revealed a significant overall effect, Dunnett's test for multiple comparisons was used to compare individual means of CRF-treated animals to controls and $t$ tests were used to compare effects in the LC to those in the cerebral aqueduct (Sinclair, 1988). Student's 2-tailed $t$ tests were also used to analyze the DHPG data.

\section{Results}

\section{Motor activity}

Test 1 . When spontaneous motor activity was measured, infusion of CRF into the cerebral aqueduct produced a significant $75 \%$ increase in ambulatory activity in photocell cages at a dose of $1 \mu \mathrm{g}$ (Fig. 1). CRF infusion into the LC did not significantly alter ambulatory activity. In contrast, infusion of CRF into the LC did significantly increase nonambulatory activity $(+81 \%)$, with the lowest effective dose being $100 \mathrm{ng}$. The increase in nonambulatory activity following CRF infusion into the LC was corroborated by observations through a 1-way mirror; CRFtreated animals generally remained in a corner of the photocell cages, constantly shifting body position for the entire $2 \mathrm{hr}$ test period, rather than actually engaging in locomotor activity.

Test 2. In the modified Porsolt swim test, CRF produced a dose-dependent decrease in floating behavior $45 \mathrm{~min}$ following infusion into the LC (Fig. 2). While this effect was seen at a 


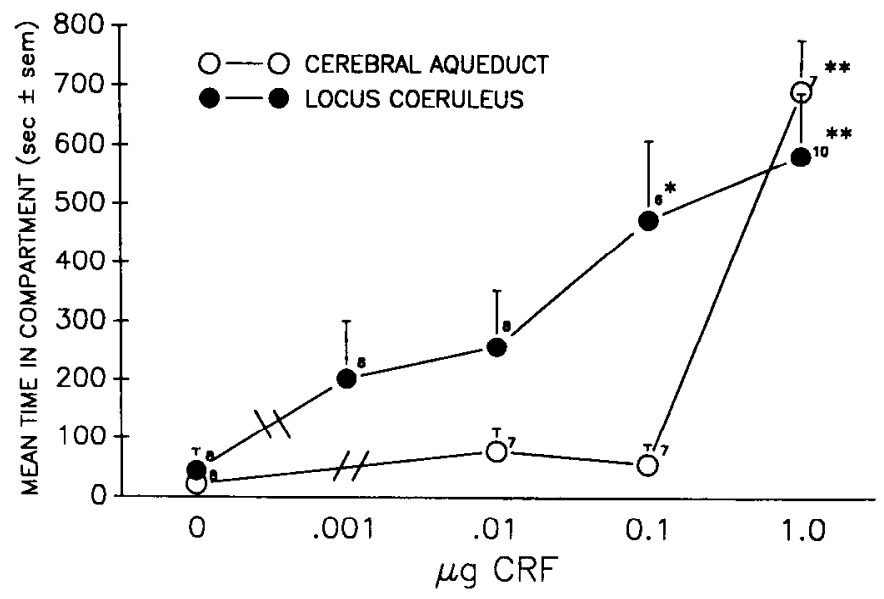

Figure 3. Time spent withdrawn in a small darkened compartment during a 15 -min test period $45 \mathrm{~min}$ following infusion of CRF into the cerebral aqueduct or locus coeruleus. The number of animals/group is indicated at each data point. Significantly different from controls using Dunnett's test, ${ }^{*} p<0.025,{ }^{* *} p<0.001$.

dose of $10 \mathrm{ng}$, a $500 \mathrm{ng}$ dose of CRF was necessary to produce a comparable decrease in floating following infusion into the lateral ventricle. Similar decreases in floating behavior were seen $10 \mathrm{~min}$ following infusion of CRF into the LC or lateral ventricle (data not shown). Struggling behavior was not affected (data not shown).

\section{Defensive-withdrawal test}

CRF produced a dose-dependent increase in time spent in the compartment $45 \mathrm{~min}$ following infusion into the LC (Fig. 3). The lowest effective dose was $100 \mathrm{ng}$. In contrast, a dose of 1 $\mu \mathrm{g}$ was necessary to produce significant effects following infusion into the cerebral aqueduct.

Assessment of open field activity in the defensive-withdrawal paradigm showed that infusion of CRF into the LC produced a dose-dependent decrease in inner and outer crossings, free and wall rearing, and exploration of the outside of the darkened compartment (Table 1). Infusion of CRF into the LC decreased the majority of these behaviors at a dose as low as $1 \mathrm{ng}$. In contrast, a dose of $1 \mu \mathrm{g}$ was necessary to produce significant effects following infusion into the cerebral aqueduct.

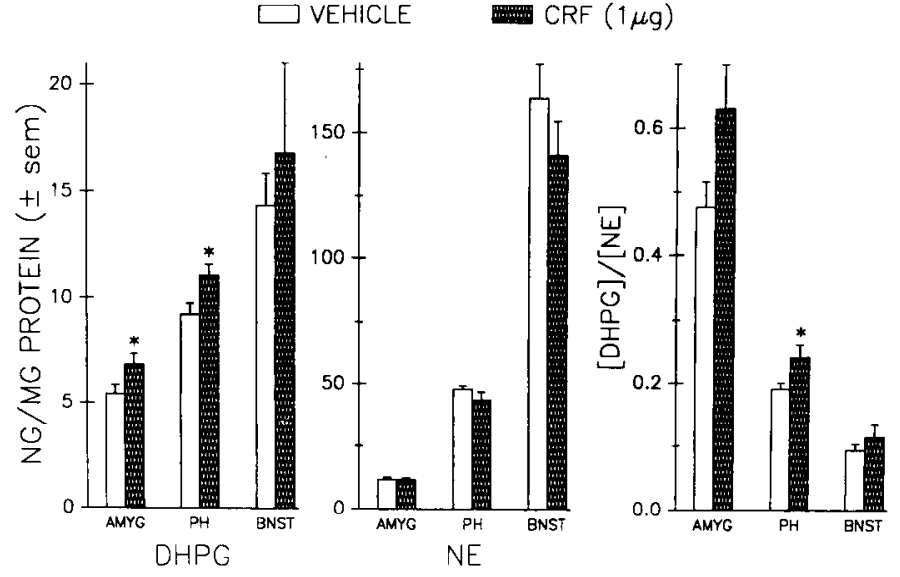

Figure 4. Concentrations of DHPG, NE, and the ratio of DHPG/NE 45 min after infusion of CRF $(1.0 \mu \mathrm{g} ; n=8)$ or vehicle $(n=9)$ into the locus coeruleus. Abbreviations: $A M Y G$, amygdala; $P H$, posterior hypothalamus; $B N S T$, bed nucleus of the stria terminalis. Significantly different from controls using Student's $t$ test, ${ }^{*} p<0.05$.

\section{$N E, D H P G, D A$, and DOPAC}

NE, DHPG, DA, and DOPAC were quantified in the amygdala, posterior hypothalamus (which includes the paraventricular nucleus), and BNST $45 \mathrm{~min}$ following infusion of CRF (1 $\mu \mathrm{g})$ or vehicle into the LC. Figure 4 shows that DHPG and NE values are high in the BNST relative to those in the amygdala and posterior hypothalamus; this is consistent with previous studies showing high NE concentrations in the BNST with a more rapid turnover in this than in other brain areas (Kilts and Anderson, 1987). Infusion of CRF into the LC produced a significant increase in the NE metabolite DHPG in the amygdala and posterior hypothalamus compared with vehicle-treated animals (Fig. 4). The DHPG/NE ratio, a further measure of NE turnover, was higher in all 3 brain areas examined, with this increase attaining statistical significance in the posterior hypothalamus. Infusion of CRF into the LC did not alter DA or DOPAC concentrations in any of the brain areas examined (data not shown).

\section{Corticosterone concentrations}

CRF produced a dose-dependent increase in plasma corticosterone concentration 45 min following infusion into the $\mathrm{LC}$ or

Table 1. Effects of CRF infusion into the locus coeruleus and cerebral aqueduct on exploration of an open field

\begin{tabular}{|c|c|c|c|c|c|c|}
\hline $\begin{array}{l}\text { Dose CRF } \\
(\mu \mathrm{g})\end{array}$ & $n$ & Crossings inner & Crossings outer & Rearing free & Rearing wall & $\begin{array}{l}\text { Exploring } \\
\text { compartment }\end{array}$ \\
\hline \multicolumn{7}{|l|}{$\mathrm{LC}$} \\
\hline 0 & 8 & $63.9 \pm 9.5$ & $157.3 \pm 21.9$ & $30.3 \pm 5.2$ & $31.1 \pm 2.3$ & $291.8 \pm 21.6$ \\
\hline 0.001 & 8 & $35.4 \pm 5.8^{*}$ & $97.8 \pm 13.9^{*}$ & $17.4 \pm 3.2^{*}$ & $18.9 \pm 3.4^{*}$ & $235.6 \pm 45.6$ \\
\hline 0.01 & 8 & $24.0 \pm 7.2^{* *}$ & $94.8 \pm 20.4^{*}$ & $12.3 \pm 3.0^{* *}$ & $25.1 \pm 5.4$ & $213.3 \pm 28.6$ \\
\hline 0.1 & 6 & $26.3 \pm 12.6^{* *}$ & $73.8 \pm 22.3^{*}$ & $11.0 \pm 5.0^{* *}$ & $14.0 \pm 4.2^{* *}$ & $93.7 \pm 28.2^{* *}$ \\
\hline 1.0 & 10 & $0.2 \pm 0.2^{* *}$ & $23.1 \pm 7.2^{* *}$ & $1.8 \pm 1.5^{* *}$ & $5.4 \pm 1.8^{* *}$ & $16.3 \pm 7.9^{* *}$ \\
\hline \multicolumn{7}{|l|}{$\mathrm{CA}$} \\
\hline 0 & 8 & $53.0 \pm 8.6$ & $140.6 \pm 10.3$ & $33.1 \pm 4.1$ & $33.8 \pm 3.2$ & $273.6 \pm 29.8$ \\
\hline 0.01 & 7 & $46.3 \pm 8.3$ & $123.0 \pm 11.0$ & $31.1 \pm 4.7$ & $25.9 \pm 3.3$ & $268.7 \pm 31.3$ \\
\hline 0.1 & 7 & $42.4 \pm 5.1$ & $131.4 \pm 11.5$ & $23.4 \pm 2.1$ & $31.9 \pm 1.1$ & $376.0 \pm 26.2$ \\
\hline 1.0 & 7 & $2.3 \pm 2.1^{* *}$ & $29.0 \pm 11.5^{* *}$ & $0.1 \pm 0.1^{* *}$ & $4.6 \pm 1.7^{* *}$ & $68.4 \pm 32.1^{* *}$ \\
\hline
\end{tabular}




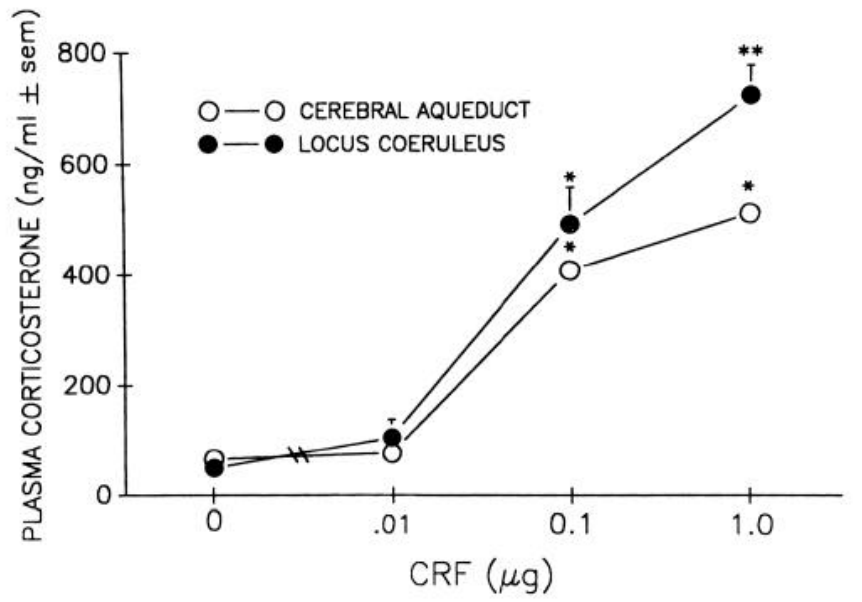

Figure 5. Plasma corticosterone concentrations $45 \mathrm{~min}$ following infusion of CRF into the cerebral aqueduct or locus coeruleus $(n=6-8$ animals in each drug group for each injection site). Significantly different from controls, ${ }^{*} p<0.001$. Significantly different from controls and cerebral aqueduct group, ${ }^{* *} p<0.01$.

cerebral aqueduct (Fig. 5). The lowest effective dose was $100 \mathrm{ng}$ for both injection sites. However, the increase in plasma corticosterone concentration was significantly greater following infusion of CRF into the LC than following infusion into the cerebral aqueduct.

\section{Histology}

A representative sample of the $\mathrm{LC}$ cannulae placement can be seen in Figure 6. It should be noted that while this figure shows some damage to the LC, the cannulae generally produced so little tissue damage that dye injections were necessary to visualize the site of injection. The percentage of correct cannulae placements for the LC, cerebral aqueduct, and lateral ventricle as 90,100 and $100 \%$, respectively. While histology could not be performed on animals used for measurement of DHPG, the high rate of correct cannulae placement in other animals indicates that these results do, in most cases, result from infusion into the LC.

\section{Discussion}

We report that direct bilateral infusion of CRF into the LC produced behavioral activating and anxiogenic effects. Behavioral activation was seen in animals adapted to photocell cages and in the modified Porsolt swim test, and anxiogenic effects were seen in a defensive-withdrawal paradigm.

Interestingly, the pattern of behavioral activation seen following infusion of CRF into the LC suggests increased arousal or agitation. For instance, infusion of CRF (100 ng) into the LC significantly increased nonambulatory spontaneous motor activity measured in photocell cages but did not alter ambulatory (i.e., locomotor) activity. Observation through a 1-way mirror corroborated these results; animals with intra-LC infusions of CRF were observed to remain in a corner of the photocell cage constantly shifting throughout the test period rather than actually engaging in locomotor activity. This pattern of spontaneous activity appears to differ from that seen following infusion of CRF into the lateral ventricle, with the latter actually appearing to increase locomotor activity. Imaki et al. (1987) found that the distance traveled by animals was increased following ICV infusion of CRF, and Kalivas and coworkers (1987) found that ICV infusion of CRF increased horizontal (i.e., locomotor) photocell activity but failed to increase vertical activity even at a dose of $20 \mu \mathrm{g}$. The increases in spontaneous locomotor activity following infusion of CRF into the LC also tend to be quite large [e.g., an $80 \%$ increase with 500 ng CRF (Cole and Koob, 1988 ) and a $280 \%$ increase with $1 \mu \mathrm{g}$ CRF (Sutton et al., 1982)], whereas infusion of CRF into the LC failed to alter locomotor activity but produced a significant $81 \%$ increase in nonlocomotor activity. Our results support those of Koob and coworkers, who found evidence suggesting that the locomotor activating effects of CRF may be mediated by forebrain rather than brainstem sites (Tazi et al., 1987), and they suggest that infusion of
Figure 6. Histological section showing location of cannulae implanted in the locus coeruleus. A hematoxylin and eosin stain was used.

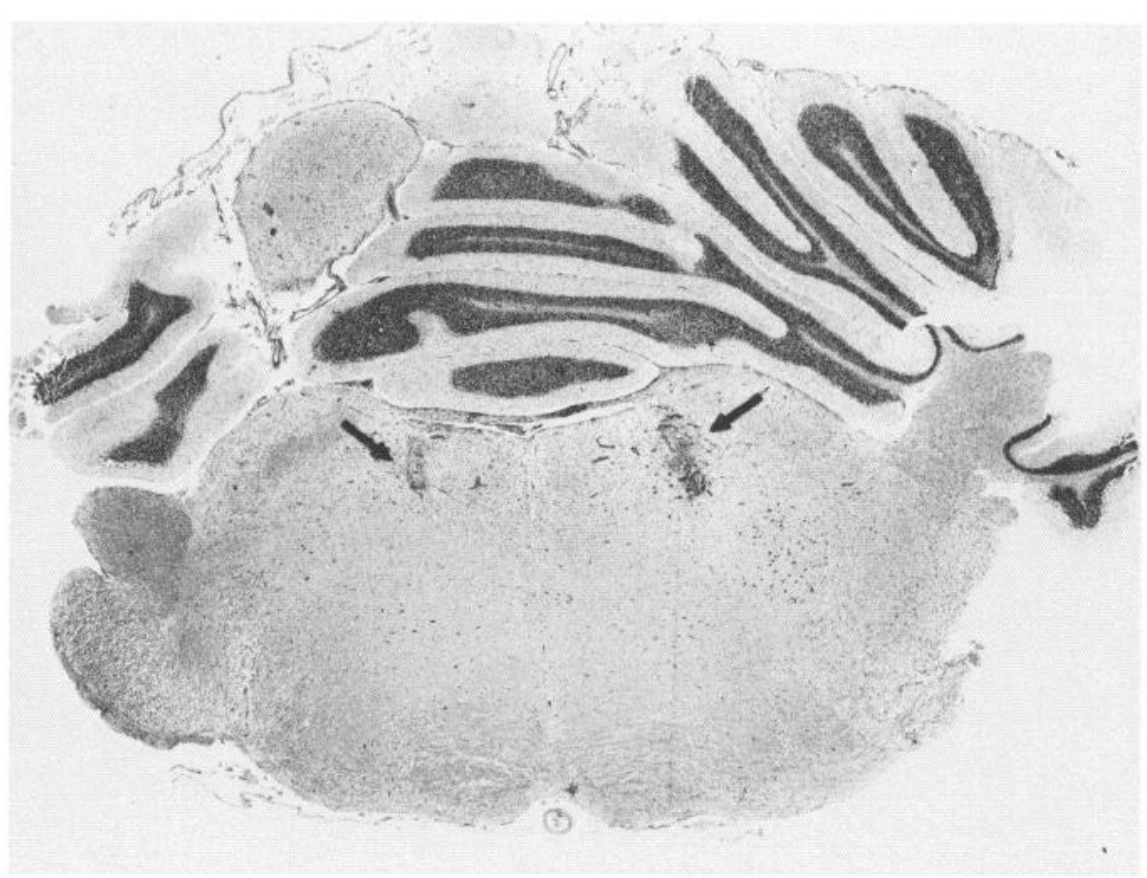


CRF into the LC may produce arousal or agitation rather than increased locomotor activity per se.

Results seen in the modified Porsolt swim test also indicate that the behavioral activating effects of CRF in the LC may be related to arousal or agitation. In the original Porsolt swim test, activity was measured in $15 \mathrm{~cm}$ of water (Porsolt et al., 1977), which allows animals to stand on the bottom of the swim tank. This results in immobility, the principal behavior these investigators were trying to measure (Porsolt et al., 1977; Hawkins et al., 1978). In contrast, the modification used in this study measured activity in $37.5 \mathrm{~cm}$ of water, making it impossible for the animals to rest their feet on the bottom of the tank (Weiss et al., 1986). Thus, arousal and agitation can be more readily assessed in a nonescapable stressful situation. Results showed that infusion of CRF into the LC produced a dose-dependent decrease in floating bchavior at a dose as low as $10 \mathrm{ng}$. This does not appear to be due to diffusion into the ventricular system; a $500 \mathrm{ng}$ dose of CRF was necessary to produce comparable effects following infusion into the lateral ventricle. Thus, infusion of CRF into the LC produced significant behavioral activation in a test that is more sensitive to arousal or agitation. These results, taken together with results of spontaneous motor activity, suggest that the behavioral activating effects of CRF may be related to arousing, stress-related effects rather than to increased locomotor activity per se.

Results in the swim test may also shed some light on CRF interactions with NE neurons in the LC. While both CRF and $\alpha_{2}$-adrenergic antagonists increase the firing rate of NE neurons in the LC (Aghajanian et al., 1977; Valentino et al., 1983; Simson and Weiss, 1987), infusion of $\boldsymbol{\alpha}_{2}$-adrenergic antagonists into the LC increases, rather than decreases, floating behavior in the modified Porsolt swim test (Weiss et al., 1986). This may be due to differential effects of these agents on NE firing patterns in the LC. Intra-LC administration of the $\alpha_{2}$-adrenergic antagonists piperoxane and yohimbine increases the firing rate of LC neurons immediately (Aghajanian et al., 1977; Simson and Weiss, 1987), whereas CRF does not do so for approximately 6-9 min, with increases lasting from 30 to 40 min (Valentino and Foote, 1988 ). Further, $\alpha_{2}$-adrenergic receptor antagonists increase sensory activated but not basal LC firing rates at all but very high doses (Simson and Weiss, 1987), whereas CRF increases basal but not stimulated LC firing rates (Valentino and Foote, 1988). Thus, behavioral results in the swim test support electrophysiological studies indicating that CRF alters the activity of NE neurons in the LC in a manner different from that of $\alpha_{2}$-adrenergic antagonists.

The fear-inducing effects of infusion of CRF into the $\mathrm{LC}$ were examined in a defensive-withdrawal paradigm recently developed by Takahashi and coworkers (1989). This paradigm makes use of species-specific behaviors seen in the wild in which rats have an initial withdrawal response to a novel or threatening environment and then later explore it (Takahashi et al., 1989). Results showed that infusion of CRF into the LC significantly increased time spent in the novel compartment and significantly decreased exploration of the open ficld. The latter effects were seen at doses as low as $1 \mathrm{ng}$, suggesting physiological relevance, and do not appear to be due to diffusion into the ventricular system because a $1000 \mathrm{ng}$ infusion of CRF into the cerebral aqueduct was necessary to comparably increase time spent in the compartment and decrease exploration of the open field. The fear-enhancement produced by infusions of low doses of CRF into the LC is of particular interest because a number of studies indicate that NE neurons in the LC are involved in fear and arousal functions. For instance, $\alpha_{2}$-adrenergic antagonists such as yohimbine, which increase NE activity in the LC, produce fear and anxiety in humans, while $\alpha_{2}$-adrenergic agonists such as clonidine, which decrease NE activity, have an anxiolytic effect (Svensson et al., 1978; Liebowitz et al., 1981). Similarly, in animal studies, electrical stimulation of the LC produces fear and anxiety in monkeys, while LC lesions have the opposite effect (Redmond et al., 1976; Redmond, 1977).

Our results in the defensive-withdrawal test may also have clinical implications in that several studies suggest that CRF hypersecretion may be involved in the pathophysiology of anxiety disorders (Roy-Byrne et al., 1986; Smith et al., 1989). While the CNS site(s) of CRF hypersecretion in anxiety disorders are not known, it is conceivable that alterations of CRF functioning in the LC may be involved in the pathophysiology of anxiety disorders in humans.

Corticosterone results support previous studies indicating that the behavioral activating and anxiogenic effects of CRF are independent of activation of the HPA axis (Eaves et al., 1985; Britton et al., 1986a, b; Takahashi et al., 1989): infusion of a $100 \mathrm{ng}$ dose of CRF into the cerebral aqueduct or LC produced similar increases in plasma corticosterone concentrations, but behavioral effects were seen at this dose only following infusion of CRF into the LC. Interestingly, infusion of a $1 \mu \mathrm{g}$ dose of CRF into the LC produced a significantly greater increase in plasma corticosterone concentration than was produced by infusion of a $1 \mu \mathrm{g}$ dose into the cerebral aqueduct. This supports neuroanatomical evidence suggesting a role for the LC in CRFinduced activation of the HPA axis. For instance, CRF-induced alterations in the activity of $\mathrm{NE}$ neurons originating from the LC could affect CRF-containing hypothalamic paraventricular nucleus neurons via a recognized NE projection from the LC to the paraventricular nucleus (Foote et al., 1983) or perhaps via LC efferents to the hippocampus, a structure which is known to regulate HPA axis function (Sapolsky et al., 1984).

In conclusion, our results indicate that the LC plays a role in the behavioral activating and fear-enhancing effects of CRF, with the bchavioral activation bcing manifested as increased arousal or agitation rather than increased locomotor activity per se. Results also showed that bilateral infusion of CRF into the LC produced significant increases in the concentration of the NE metabolite DHPG in several forebrain projection areas of the LC (i.e., the posterior hypothalamus and amygdala). These data, taken together, suggest that CRF may produce its behavioral activating and anxiogenic effects, at least in part, by increasing activity of LC NE neurons.

\section{References}

Aghajanian, G. K., J. M. Cedarbaum, and R. Y. Wang (1977) Evidence for norepinephrine-mediated collateral inhibition of locus coeruleus neurons. Brain Res. 136: 570-577.

Braestrup, C., M. Nielson, and J. Scheel-Kruger (1974) Accumulation and disappearance of noradrenaline and its major metabolites synthesized from intraventricularly injected ${ }^{3} \mathrm{H}$-dopamine in the rat brain. J. Neurochem. 23: 569-578.

Britton, D. R., G. F. Koob, J. Rivier, and W. Vale (1982) Intraventricular corticotropin-releasing factor enhances behavioral effects of novelty. Life Sci. 31:363-367.

Britton, K. T., J. Morgan, J. Rivier, W. Vale, and G. F. Koob (1985) Chlordiazepoxide attenuates response suppression induced by corticotropin-releasing factor in the conflict test. Psychopharmacology 86: 170-174.

Britton, D. R., M. Varela, A. Garcia, and M. Rosenthal (1986a) Dexa- 
methasone suppresses pituitary-adrenal but not behavioral effects of centrally administered CRF. Life Sci. 38: 211-216.

Britton, K. T., G. Lee, R. Dana, S. C. Risch, and G. F. Koob (1986b) Activating and "anxiogenic" effects of corticotropin releasing factor are not inhibited by blockade of the pituitary-adrenal system with dexamethasone. Life Sci. 39: 1281-1286.

Brown, M. R., and L. A. Fisher (1985) Corticotropin-releasing factor: Effects on the autonomic nervous system and visceral systems. Fed. Proc. 44: 243-248.

Chappell, P. B., M. A. Smith, C. D. Kilts, G. Bissette, J. Ritchie, C. Anderson, and C. B. Nemeroff (1986) Alterations in corticotropinreleasing factor-like immunoreactivity in discrete rat brain regions after acute and chronic stress. J. Neurosci. 6: 2908-2914.

Cole, B. J., and G. F. Koob (1988) Propranolol antagonizes the enhanced conditioned fear produced by corticotropin releasing factor. J. Pharmacol. Exp. Ther. 247: 902-910.

Dunn, A. J., and C. W. Berridge (1987) Corticotropin-releasing factor administration elicits a stress-like activation of cerebral catecholaminergic systems. Pharmacol. Biochem. Behav. 27: 685-691.

Dunn, A. J., and S. E. File (1987) Corticotropin-releasing factor has an anxiogenic action in the social interaction test. Horm. Behav. 21: 193-202.

Eaves, M., K. Thatcher-Britton, J. Rivier, W. Vale, and G. F. Koob (1985) Effects of corticotropin-releasing factor on locomotor activity in hypophysectomized rats. Peptides $6:$ 923-926.

Foote, S. L., F. E. Bloom, and G. Aston-Jones (1983) Nucleus locus coeruleus: New evidence of anatomical and physiological specificity. Physiol. Rev. 63: 844-914.

Glavin, G. B. (1985) Stress and brain noradrenaline: A review. Neurosci. Biobehav. Rev. 9: 233-243.

Hawkins, J., R. Hicks, N. Phillips, and R. D. Moore (1978) Swimming rats and human depression. Nature 274: 512.

Heffner, T. G., J. A. Hartman, and L. S. Seiden (1980) A rapid method for the regional dissection of the rat brain. Pharmacol. Biochem. Behav. 13: 453-456.

Imaki, T., T. Shibasaki, A. Masuda, H. Demura, K. Shizume, and N. Ling (1987) Effects of adrenergic blockers on corticotropin-releasing factor-induced behavioral changes in rats. Regul. Peptides 19: 243252.

Kalivas, P. W., P. Duffy, and L. G. Latimer (1987) Neurochemical and behavioral effects of corticotropin-releasing factor in the ventral tegmental area of the rat. J. Pharmacol. Exp. Ther. 242: 757-763.

Kilts, C. D., and C. M. Anderson (1987) Mesoamygdaloid dopamine neurons: Differential rates of dopamine turnover in discrete amygdaloid nuclei of rat brain. Brain Res. 416: 402-408.

Korf, J., G. K. Aghajanian, and R. H. Roth (1973) Increased turnover of norepinephrine in the rat cerebral cortex during stress: Role of the locus coeruleus. Neuropharmacology 12: 933-938.

Li, P. P., J. J. Warsh, and D. D. Godse (1981) 3,4-Dihydroxyphenylethyleneglycol (DHPG) formation: The major route of rat brain norepinephrine metabolism. Prog. Neuro-Psychopharmacol. 5: 531-535.

Li, P. P., J. J. Warsh, and D. D. Godse (1983) Rat brain norepinephrine metabolism: Substantial clearance through 3,4-dihydroxyphenylethyleneglycol formation. J. Neurochem. 41: 1065-1071.

Liebowitz, M. R., A. J. Fyer, P. McGrath, and D. F. Klein (1981) Clonidine treatment of panic disorder. Psychopharmacol. Bull. 17: 122-123.

Lowry, O. H., N. J. Rosebrough, A. L. Farr, and R. J. Randall (1951) Protein measurement with the Folin phenol reagent. J. Biol. Chem. 193: 265-275.

Merchenthaler, I. (1984) Corticotropin releasing factor (CRF)-like immunoreactivity in the rat central nervous system. Extrahypothalamic distribution. Peptides 5: 53-69.

Morley, J. E., and A. S. Levine (1982) Corticotropin releasing factor, grooming and ingestive behavior. Life Sci. 31: 1459-1464.

Murphy, B. E. P. (1967) Some studies of the protein binding of steroids and their application to the routine micro and ultramicro measurement of various steroids in body fluids by competitive protein binding radioassay. J. Clin. Endocrinol. Metab. 27: 973-990.

Porsolt, R. D., M. Le Pichon, and M. Jalfre (1977) Depression: A new animal model sensitive to antidepressant treatments. Nature 226: 730-732.

Redmond, D. E., Jr. (1977) Alterations in the functions of the nucleus locus coeruleus: A possible model for studies of anxiety. In Animal
Models in Psychiatry and Neurology, I. Hanin and E. Usdin, eds., pp. 293-303, Pergamon, New York.

Redmond, D. E., Jr., Y. M. Huang, D. R. Synder, and J. W. Maas (1976) Behavioural effects of stimulation of the nucleus coeruleus in the stump-tailed monkey Macaca arctoides. Brain Res. 116: 502-510.

Ritchie, J. C., B. J. Carroll, P. R. Olton, V. Shively, and M. Feinberg (1985) Plasma cortisol determination for the dexamethasone suppression test. Arch. Gen. Psychiatry 42: 493-497.

Rivier, C., M. Brownstein, J. Spiess, J. Rivier, and W. Vale (1982) In vivo corticotropin-releasing factor-induced secretion of adrenocorticotropin, beta-endorphin, and corticosterone. Endocrinology 110:272278.

Roy-Byrne, P. P., T. W. Uhde, R. M. Post, W. Gallucci, G. P. Chrousos, and P. W. Gold (1986) The corticotropin-releasing hormone stimulation test in patients with panic disorder. Am. J. Psychiatry 143: 896-899.

Sapolsky, R. M., L. C. Krey, and B. S. McEwen (1984) Stress downregulates corticosterone receptors in a site-specific manner in the brain. Endocrinology 114: 287-292

Scatton, B. (1982) Brain 3,4-dihydroxyphenylethyleneglycol levels are dependent on central noradrenergic neuron activity. Life Sci. 31:495504.

Simson, P. E., and J. M. Weiss (1987) Alpha-2 receptor blockade increases responsiveness of locus coeruleus neurons to excitatory stimulation. J. Neurosci. 7: 1732-1740.

Sinclair, J. D. (1988) Multiple t-tests are appropriate in science. Trends Pharmacol. Sci. 9: 12-13.

Sirinathsinghji, D. J. S., L. H. Rees, J. Kivier, and W. Vale (1983) Corticotropin-releasing factor is a potent inhibitor of sexual receptivity in the female rat. Nature 305: 230-235.

Skoog, K. M., S. T. Cain, and C. B. Nemeroff (1986) Centrally administered neurotensin suppresses locomotor hyperactivity induced by d-amphetamine but not by scopolamine or caffeine. Neuropharmacology 25: 777-782.

Smith, M. A., J. Davidson, J. C. Ritchie, H. Kudler, S. Lipper, P. Chappell, and C. B. Nemeroff (1989) The corticotropin-releasing hormone test in patients with post-traumatic stress disorder. Biol. Psychiatry 26: 349-355.

Sutton, R. E., G. F. Koob, M. LeMoal, R. Rivier, and W. Vale (1982) Corticotropin releasing factor produces behavioural activation in rats. Nature 297: 331-333.

Svensson, T.H. (1987) Peripheral, autonomic regulation of locus coeruleus noradrenergic neurons in brain: Putative implications for psychiatry and psychopharmacology. Psychopharmacology 92: 1-7.

Svensson, T. H., R. Persson, L. Wallin, and J. Walinder (1978) Anxiolytic action of clonidine. Nord. Psyk. Tidskr. 32: 439-441.

Swanson, L. W., P. E. Sawchenko, J. Rivier, and W. W. Vale (1983) Organization of ovine corticotropin-releasing factor immunoreactive cells and fibers in the rat brain: An immunohistochemical study. Neuroendocrinology 36: 165-186.

Swerdlow, N. R., M. A. Geyer, W. W. Vale, and G. F. Koob (1986) Corticotropin-releasing factor potentiates acoustic startle in rats: Blockade by chlordiazepoxide. Psychopharmacology 88: 147-152.

Takahashi, L. K., N. H. Kalin, J. A. Vanden Burgt, and J. E. Sherman (1989) Corticotropin-releasing factor modulates defensive-withdrawal and exploratory behavior in rats. Behav. Neurosci. (in press)

Tazi, A., N. R. Swerdlow, M. LeMoal, J. Rivier, W. Vale, and G. F. Koob (1987) Behavioral activation by CRF: Evidence for the involvement of the ventral forebrain. Life Sci. 41: 41-49.

Thierry, A., F. Javoy, J. Glowinski, and S. Kety (1968) Effects of stress on the metabolism of norepinephrine, dopamine, and serotonin in the central nervous system of the rat. I. Modification of norepinephrine turnover. J. Pharmacol. Exp. Ther. 163: 163-171.

Uphouse, L. L., C. B. Nemeroff, G. A. Mason, A. J. Prange, Jr., and S. C. Bondy (1982) Interactions between "handling" and acrylamide on endocrine response in rats. Neurotoxicology 3: 121-125.

Vale, W., J. Spiess, C. Rivier, and J. Rivier (1981) Characterization of a 41-residue ovine hypothalamic peptide that stimulates secretion of corticotropin and $\beta$-endorphin. Science 213: 1394-1397.

Valentino, R. J., and S. L. Foote (1988) Corticotropin-releasing hormone increases tonic but not sensory-evoked activity of noradrenergic locus coeruleus neurons in unanesthetized rats. J. Neurosci. 8: 10161025 .

Valentino, R. J., S. L. Foote, and G. Aston-Jones (1983) Corticotropin- 
releasing factor activates noradrenergic neurons of the locus coeruleus. Brain Res. 270: 363-367.

Warsh, J. J., P. P. Li, D. D. Godse, and S. Cheung (1981) Brain noradrenergic neuronal activity affects 3,4-dihydroxyphenylethyleneglycol (DHPG) levels. Life Sci. 29: 1303-1307.
Weiss, J. M., P. G. Simson, L. J. Hoffman, M. J. Ambrose, S. Cooper, and $A$. Webster (1986) Infusion of adrenergic receptor agonists and antagonists into the locus coeruleus and ventricular system of the brain. Neuropharmacology 25: 367-384. 